\title{
MicroRNA-449a delays lung cancer development through inhibiting KDM3A/HIF-1a axis
}

\author{
Shan Hu, Peng Cao, Kangle Kong, Peng Han, Yu Deng, Fan Li ${ }^{*}$ and Bo Zhao*
}

\begin{abstract}
Background: It has been established that microRNA (miR)-449a is anti-tumorigenic in cancers, including lung cancer. Therefore, this study further explored miR-449a-mediated mechanism in lung cancer, mainly focusing on lysine demethylase 3A/hypoxia-induced factor-1 a (KDM3A/HIF-1a) axis.

Methods: miR-449a, KDM3A and HIF-1 a levels in lung cancer tissues and cell lines (A549, H1299 and H460) were measured. Loss- and gain-of-function assays were performed and then cell proliferation, cell cycle, apoptosis, invasion and migration were traced. The relationship between KDM3A, miR-449a and HIF-1a was verified. Tumor growth in vivo was also monitored.

Results: Both lung cancer tissues and cells exhibited reduced miR-449a and raised KDM3A and HIF-1a levels. miR449a interacted with KDM3A; HIF-1 a could bind with KDM3A. Up-regulating miR-449a hindered while suppressing miR-449a induced lung cancer development via mediating HIF-1a. Elevating KDM3A promoted cellular aggression while down-regulating KDM3A had the opposite effects. Up-regulating KDM3A or HIF-1a negated up-regulated miR449a-induced effects on cellular growth in lung cancer. Restoring miR-449a impaired tumorigenesis in vivo in lung cancer.
\end{abstract}

Conclusion: It is eventually concluded that miR-449a delays lung cancer development through suppressing KDM3A/ HIF-1a axis.

Keywords: Lung cancer, MicroRNA-449a, Lysine demethylase 3A, Hypoxia-induced factor-1a

\section{Background}

Lung cancer is the major human malignancy, mainly composed of small cell lung cancer and non-small cell lung cancer (NSCLC) [1]. It is usually diagnosed as advanced in which lung cancer cells quickly pass through the lymph or blood and spread to other organs, causing a high mortality globally [2]. Statistically, more than twice as many men as women have been diagnosed with lung

*Correspondence: Lifan56456@outlook.com; ZhaoBoZBzb1997@outlook. com

Department of Thoracic Surgery, Tongji Hospital, Tongji Medical College, Huazhong University of Science and Technology, Qiaokou District, No.

1095 Jiefang Avenue, Qiaokou District, Wuhan 430030, Hubei, China cancer worldwide, which is mainly due to the risk factor, smoking [3]. Most lung cancer patients, especially those in an advanced stage, have poor prognosis due to delayed diagnosis and poor response to conventional treatments [4]. Though received treatments, up to $15 \%$ of lung cancer patients develop second primary lung cancer [5]. At present, the pathogenesis of lung cancer is not completely comprehended that asks for in-depth explorations.

Dysregulated non-coding RNAs, including microRNAs (miRNAs) can affect the occurrence and metastasis of lung cancer through transcriptional, post transcriptional and epigenetic activities [6]. miR-449a is a part in regulating lung cancer proliferation, migration, invasion and epithelial to mesenchymal transition [7]. Clinically 
analyzed, low expression of miR-449a is closely connected with differentiation, clinical staging and lymph node metastasis of lung cancer patients [8]. In treating lung cancer, miR-449a has the potential to overcome the resistance of epidermal growth factor receptor tyrosine kinase inhibitors [9]. Also, targeting miR-449a could sensitize non-small sell lung cancer cells to ionizing radiation, thereby suppressing tumor growth [10]. Lysine demethylase 3A (KDM3A), also known as JMJD1A, is the overexpressed gene in lung cancer [11]. KDM3A is the vital modulator for biological functions of NSCLC cancer cells [12]. Mechanistically, KDM3A could induce the production of inhibitory cytokines in lung adenocarcinoma cells, thereby promoting immune escape of cancer cells [13]. Serving as a hypoxic response gene, KDM3A could be mediated by hypoxia-inducible factor- $1 \alpha$ (HIF-1 $\alpha$ ), the key gene in lung cancer under a hypoxic microenvironment [14]. The stability of HIF- $1 \alpha$ has been recognized to modulate the stem-like characteristics of lung cancer cells [15], as well as drug resistance and tumor progression in lung cancer [16]. Regarding to the functional roles of miR-449a, KDM3A and HIF- $1 \alpha$ in lung cancer, this research was initiated to decode whether their integrity involved in lung cancer and targeting miR$449 \mathrm{a} / \mathrm{KDM} 3 \mathrm{~A} / \mathrm{HIF}-1 \alpha$ axis regulated cancer progression.

\section{Methods and materials}

\section{Ethics statement}

Approved by the ethics committee of Tongji Hospital, Tongji Medical College, Huazhong University of Science and Technology, this study was conducted following Declaration of Helsinki. An signed consent was provided by each participant. Animal treatment was humanely performed.

\section{Sample collection}

Lung cancer tissues and normal tissues were harvested from 80 patients (not received radiotherapy or chemotherapy) in Tongji Hospital, Tongji Medical College, Huazhong University of Science and Technology from April 2018 to March 2019. All tissues were frozen in liquid nitrogen. The diagnosis was conducted by the pathologists in Tongji Hospital, Tongji Medical College, Huazhong University of Science and Technology in compliance with the principles of the latest classification regulations of the World Health Organization [17].

\section{Cell culture}

Human lung cancer cell lines (A549, H1299 and H460) and normal human lung epithelial cell line (BEAS-2B) were supplied by the Cell Bank of Chinese Academy of Sciences (Shanghai, China) and maintained in 10\% fetal bovine serum (FBS)-Dulbecco's modified Eagle medium (DMEM) (both from HyClone, Los Angeles, USA) [18].

\section{Cell transfection}

Based on the known miR-449a and KDM3A sequences in NCBI, plasmids were constructed by Sangon Biotech (Shanghai, China). Cells of passage 3 were trypsinized and cultured into a monolayer in a 24-well plate at $1 \times 10^{3}$ cells/well. Cells at $75 \%$ confluence were transfected with miR-449a-mimic/inhibitor, shKDM3A, overexpression (oe)-KDM3A, miR-449amimic + oe-HIF-1 $\alpha, \quad$ miR-449a-mimic + oe-KDM3A, or their corresponding negative control (NC) through Lipofectamine 2000 (Thermo Fisher Scientific, Waltham, USA) [19].

\section{Cell counting kit (CCK)-8 assay}

Cells in a 96-well plate $\left(1 \times 10^{3}\right.$ cells/well $)$ were cultured for $0,24,48$, and $72 \mathrm{~h}$, respectively. At each time point, each well was supplemented with $10 \mu \mathrm{L}$ CCK-8 reagent (Dojindo, Kumamoto, Japan). Cells that were continuously cultured for further $3 \mathrm{~h}$ were detected by a microplate reader (Infinite M200 PRO, TECAN, Switzerland) to measure the optical density $\left(\mathrm{OD}_{450 \mathrm{~nm}}\right)[18]$.

\section{Transwell assay}

Cells $\left(6 \times 10^{4}\right.$ cells) were resuspended in serum-free DMEM $(200 \mu \mathrm{L})$, and added in the chamber. An attractant was set with $10 \%$ FBS-DMEM $(600 \mu \mathrm{L})$ in the lower chamber. Cells were incubated for $24 \mathrm{~h}$ in migration assay and for $48 \mathrm{~h}$ in invasion assay. Cells that migrated or invaded were fixed with $4 \%$ paraformaldehyde, followed by staining with $0.1 \%$ crystal violet and photography by a microscope (TE2000; Nikon, Tokyo, Japan) in at least five random fields of view [20].

\section{Flow cytometry}

A549 cells transfected for $48 \mathrm{~h}$ were pre-cooled with 70\% frozen ethanol and treated with propidium iodide (PI) and RNase (BD Company, New Jersey, USA). Cell cycle distribution was analyzed with a flow cytometer (BD Biosciences, New Jersey, USA) [21]. For cell apoptosis, cells were detached with $0.25 \%$ trypsin, added with Roswell Park Memorial Institute-1640 medium containing 10\% FBS, centrifuged at $1000 \mathrm{r} / \mathrm{min}$, fixed with $70 \%$ ethanol and adjusted to $1 \times 10^{6}$ cells $/ \mathrm{mL}$. The cell suspension was added with $10 \mathrm{~mL}$ Annexin V-fluorescein isothiocyanate (FITC)/PI $(556,547$, Shanghai Shuojia Biotechnology Co., Ltd., China) and cell apoptosis was detected in a flow cytometer (XL type, Conlter, USA). Excitation 
wavelength was $480 \mathrm{~nm}$, FITC was detected at $530 \mathrm{~nm}$ and PI was detected at greater than $575 \mathrm{~nm}$. The apoptotic rate was percentage of apoptotic cells in total cells [22].

\section{Reverse transcription quantitative polymerase chain reaction (RT-qPCR)}

RNA was extracted from tissues and cells by Trizol (Life Technologies, MD, USA). cDNA was generated by a reverse transcription kit (Thermo, Massachusetts, USA). miR-449a, KDM3A, and HIF-1 $\alpha$ levels were measured by SYBR Green method on the ABI7500 fluorescent quantitative PCR instrument, and normalized to U6 and $\beta$-actin. Data analysis was conferred to $2^{-\Delta \Delta \mathrm{Ct}}$ method. Primers were found in Table 1 [23].

\section{Western blot assay}

The extracted total protein in cells and tissues by radioimmunoprecipitation assay (RIPA) buffer was quantified by bicinchoninic acid protein assay kit (Thermo Fisher Scientific). Subsequently, total protein was separated with $10 \%$ sodium dodecyl sulfate-polyacrylamide gel electrophoresis and transferred onto a $0.45-\mu \mathrm{M}$ polyvinylidene fluoride membrane (MilliPore, MA, USA). The protein membrane incubated with the specific antibodies were developed by enhanced chemiluminescence and exposed by Image Quant LAS 4000C (GE Company, USA). Primary antibodies included KDM3A $(1: 200,12,835-1$-AP, Proteintech), HIF-1 $\alpha$ (1:1000, 610,958, BD Biosciences), Cleaved-PARP (ab32064, Abcam, MA, USA), CleavedCASP3 (9661, Cell Signaling Technology) and $\beta$-actin (1:1000, sc-47778, Santa Cruz Biotechnology) while secondary antibody included anti-rabbit immunoglobulin G (IgG; 7074, 1:2000, Cell Signaling Technology). $\beta$-actin was the internal control [24].

\section{Dual luciferase reporter gene assay}

The binding sites of miR-449a and KDM3 were firstly analyzed by RNA22 and their targeting relation was validated by dual luciferase reporter gene experiment. KDM3A mRNA 3'untranslated region (3'UTR) and the mutant sequence were cloned into the pGL3 dual luciferase reporter gene vector (Promega, WI, USA) to construct wild-type and mutant KDM3A 3'UTR vectors. The constructed vectors with miR-449a mimic/NCmimic were co-transfected into A549 cells through Lipofectamine 2000 (Invitrogen). The luciferase activity was measured by the dual luciferase reporter system (Glo$\max 20 / 20$, ATCC, Manassas, VA, USA) [25].

\section{Co-immunoprecipitation (Co-IP) assay}

Cells were lysed with IP lysis buffer $(500 \mu \mathrm{L})$ on ice and centrifuged at $13,500 \mathrm{r} / \mathrm{min}$. The supernatant was reacted with the antibody overnight, added with protein A + G agarose (P2055, Beyotime, China) and centrifuged at 13,500 r/min. After washing 3 times with cold lysis buffer, the immunoprecipitate was combined with sodium dodecyl sulfate, heated to $98{ }^{\circ} \mathrm{C}$ and treated with sodium dodecyl sulphate polyacrylamide gel electrophoresis. After that, the protein was transferred to a $0.22-\mu \mathrm{m}$ polyvinylidene fluoride membrane, immersed in $10 \%$ skimmed milk and combined with the primary antibodies KDM3A (1:200, 12,835-1-AP, Proteintech) and HIF- $1 \alpha$ (1:1000, 610,958, BD Biosciences). Then, the membrane was washed 3 times with Tris-buffered saline with Tween-20 and cultured with IgG antibody (7074, 1:2000, Cell Signaling Technology). The pellet was rinsed with RIPA buffer and then subjected to Western blot analysis. Normal IgG was used as a NC [26].

\section{Tumor xenografts in nude mice}

Twenty-five BALA/C nude mice (4-6 weeks old, 18-20 g) from Hunan SJA laboratory Animal Co., Ltd. (Changsha, China) were subcutaneously injected with A549 cells $\left(1 \times 10^{3}\right.$ cells $)$ into the right armpit. Phosphate-buffered saline (PBS)-treated A549 cells were used as NC. The average diameter of tumors was measured weekly. After 8 $\mathrm{w}$, all mice were euthanized and tumor weight was measured [27].

Table 1 Primer sequences

\begin{tabular}{lll}
\hline Primer sequences & Forward $\left(\mathbf{5}^{\prime} \rightarrow \mathbf{3}^{\prime}\right)$ & Reverse $\left(\mathbf{5}^{\prime} \boldsymbol{\rightarrow} \mathbf{3}^{\prime}\right)$ \\
\hline miR-449a & CTCGCTGGCAGTGTATTGTTAG & TATCGTTGTACTCCAGACCAAGAC \\
KDM3A & AACTATTGAGCCACACAGACAGG & ACACATACTCCAAACCCACACC \\
HIF-1a & GGTTCCAGCAGACCCAGTTA & AGGCTCCTTGGATGAGCTTT \\
U6 & CTCGCTTCGGCAGCACA & AACGCTTCACGAATTTGCGT \\
$\beta$-actin & CATCACCATCTTCCAGGAGCG & TGACCTTGCCCACAGCCTTG
\end{tabular}

miR-449a microRNA-449a, KDM3A Lysine demethylase 3A, HIF-1a Hypoxia-induced factor-1a 


\section{Statistical analysis}

The independent t-test was applied to compare data between two groups. All data were analyzed by statistical software SPSS 17.0 (SPSS, Chicago, USA) and expressed as mean \pm standard deviation. $P<0.05$ was considered a significant difference.

\section{Results}

\section{Down-regulated miR-449a is manifested in lung cancer tissues and cells}

miR-449a expression has been measured to reduce in primary lung cancer [28]. Similar to that we applied RT-qPCR to detect miR-449a level in lung cancer and ultimately discovered that miR-449a was lowly expressed in lung cancer tissues and cells (Additional file 1: Fig. S1a, b). Among lung cancer cell lines, lowest miR-449a level was detected in A549 and H1299 cells, thus A549 and H1299 cells were used miR-449a overexpression experiments.

\section{Over-expressing miR-449a delays lung cancer development while down-regulating miR-449a has the opposite effects on lung cancer}

To further clarify the effect of miR-449a on lung cancer, cell proliferation, apoptosis/cell cycle distribution, migration and invasion, as well as apoptosis-related proteins (Cleaved-PARP and Cleaved CASP3) were examined by CCK-8, flow cytometry, Transwell assay and western blot, respectively. The experimental results demonstrated that in response to up-regulation of miR-449a in A549 (Additional file 1: Fig. S1c), proliferation, migration and invasion capacities were impaired, apoptosis was enhanced, increased cells arrested in G0/G1 phase, and CleavedPARP and Cleaved CASP3 protein expression were elevated (Fig. 1a-f).

To make the research results more rigorous, we repeated the above experiments in another lung cancer cell line $\mathrm{H} 1299$, and the results were similar to those in A549 cells (Additional file 3: Fig. S3). Moreover, we performed miR449a down-regulation assay A549 cells (Additional file 1: Fig. S1d), and found that down-regulating miR-449a enhanced the growth of A549 cells (Fig. 2a-f).

HIF- $1 \alpha$, generally highly expressed in lung cancer cells, is considered as the main mediator of lung cancer cells under hypoxia $[29,30]$. We determined HIF-1 $\alpha$ level in lung cancer tissues and normal tissues and tested a higher level of
HIF-1 $\alpha$ in lung cancer tissues (Additional file 2: Fig. S2a). HIF- $1 \alpha$ level presented the same trend in lung cancer cells in vitro (Additional file 2: Fig. S2b). Subsequently, the effect of miR-449a on HIF- $1 \alpha$ was explored, and the result indicated that overexpressing miR-449a reduced HIF- $1 \alpha$ level (Additional file 2: Fig. S2c) while silencing miR-449a raised HIF-1 $\alpha$ expression in cells (Additional file 2: Fig. S2d).

\section{Enhanced KDM3A level in lung cancer cells promotes cellular aggression}

KDM3A is the mediator of biological progress in cancers and is up-regulated in lung cancer $[13,31]$. We also tested KDM3A expression in lung cancer tissues and cells, and found that KDM3A showed an increase in both lung cancer tissues and cells (Additional file 1: Fig. S1e, f). To study the effects of KDM3A in lung cancer, we transfected oe-KDM3A and sh-KDM3A plasmids into A549 cells to up-regulate or down-regulate KDM3A expression (Additional file 1: Fig. S1g). Then, we observed cellular growth and tested that cell growth was promoted after oe-KDM3A treatment while suppressed after sh-KDM3A treatment (Fig. 3a-f). Shortly, up-regulating KDM3A promoted while down-regulating KDM3A suppressed the development of lung cancer.

\section{miR-449a interacts with KDM3A; HIF-1 a could bind to KDM3A}

miR-449a and KDM3A have been recorded to regulate lung cancer, respectively $[17,29]$. Focused on the issue that whether KDM3A was involved in the process of miR-449a regulating lung cancer, we firstly determined the effect of miR-449a on KDM3A expression and found that overexpressing miR-449a decreased KDM3A level in cells (Fig. 4a). After that, the targeting relation was analyzed between miR-449a and KDM3A on Jefferson website (Fig. 4b) and validated by dual luciferase reporter experiment. It turned out that miR-449a-mimic weakened luciferase activity of WTKDM3A (Fig. 4c).

HIF1 $\alpha$ and KDM3A are positively correlated [32, 33]. However, how KDM3A regulates the expression of HIF $1 \alpha$ is still unclear. Thus, we conducted assays to explore the interaction between the two. First,

\footnotetext{
(See figure on next page.)

Fig. 1 Over-expressing miR-449a delays A549 cell growth. a CCK-8 assay analyzed A549 cell proliferation after overexpression of miR-449a; b Flow cytometry analyzed A549 cell cycle after overexpression of miR-449a; c Western blot analyzed the expression of apoptosis-related proteins in A549 cells after overexpression of miR-449a; d Flow cytometry analyzed A549 cell apoptosis after overexpression of miR-449a; e Transwell assay analyzed A549 cell migration after overexpression of miR-449a; f Transwell assay analyzed A549 cell invasion after overexpression of miR-449a; The data from three independent experiments were expressed as mean \pm standard deviation. ${ }^{*} P<0.05$ compared with the NC-mimic group
} 


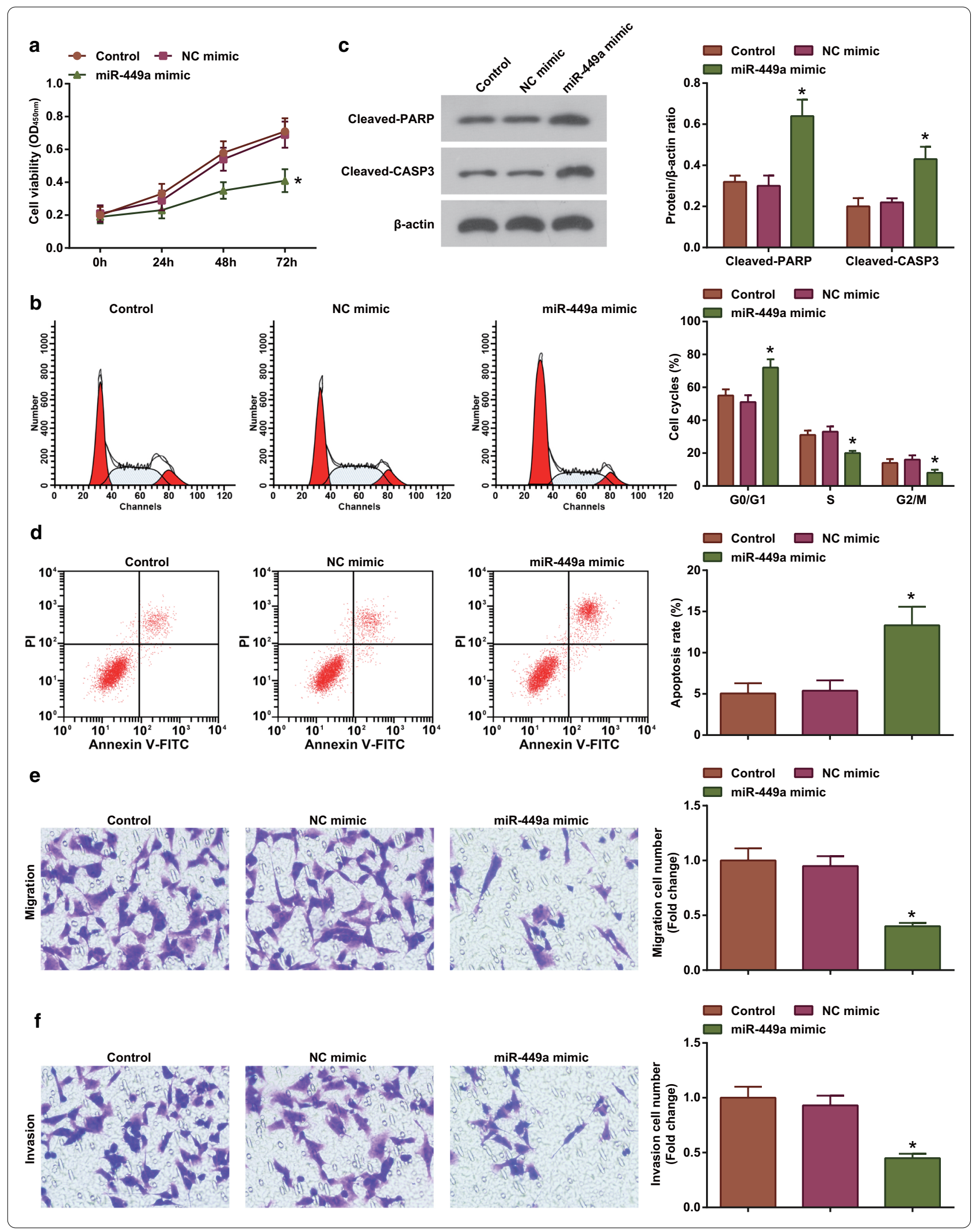




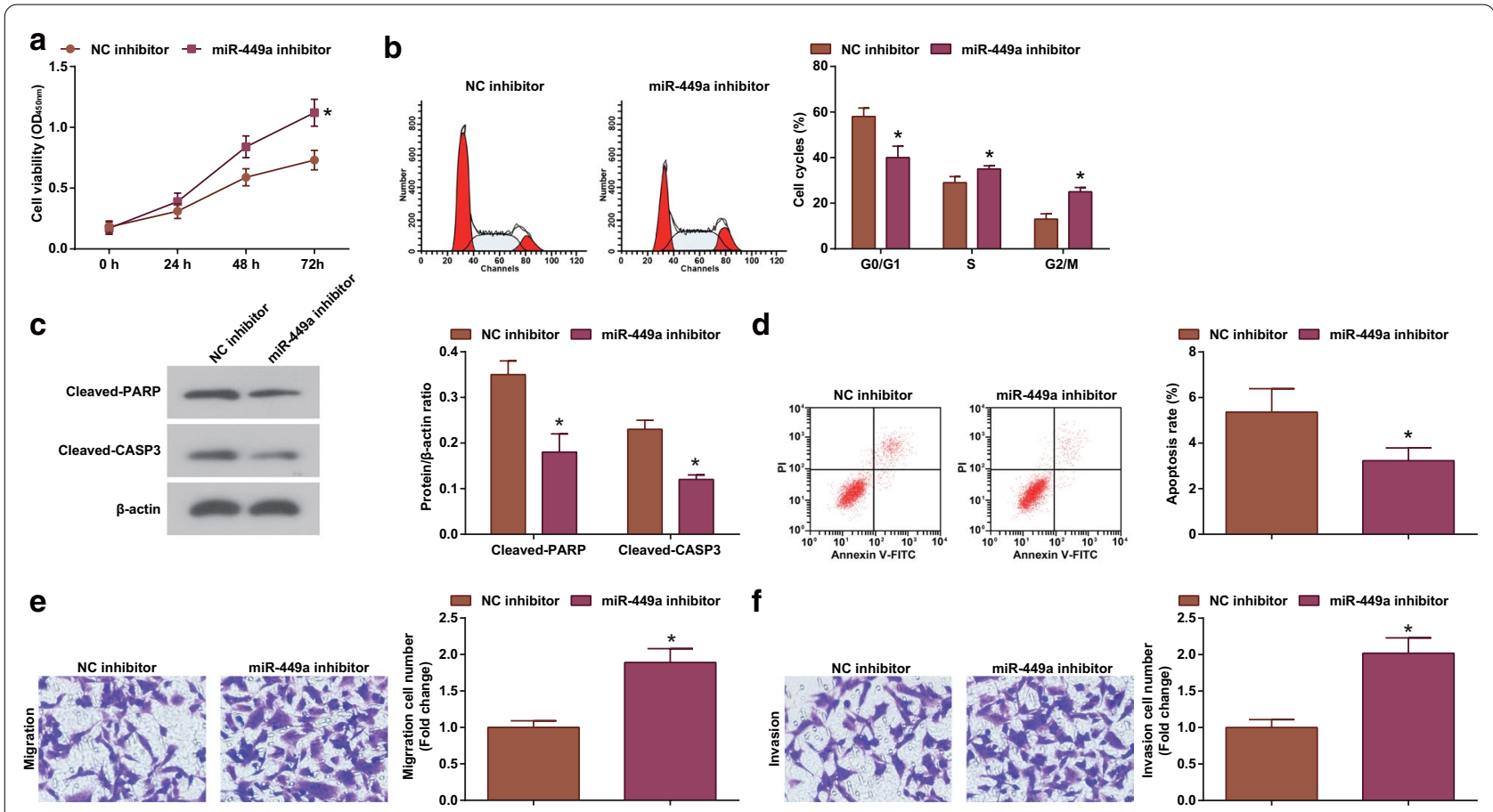

Fig. 2 Suppressing miR-449a accelerates lung cancer development. a CCK-8 assay analyzed A549 cell proliferation after inhibiting miR-449a; b Flow cytometry analyzed A549 cell cycle after inhibiting miR-449a; c Western blot analyzed the expression of apoptosis-related proteins in A549 cells after inhibiting miR-449a; d Flow cytometry analyzed A549 cell apoptosis after inhibiting miR-449a; e Transwell assay analyzed A549 cell migration after inhibiting miR-449a; $\mathbf{f}$ Transwell assay analyzed A549 cell invasion after inhibiting miR-449a; The data from three independent experiments were expressed as mean \pm standard deviation. ${ }^{*} P<0.05$ compared with the NC-inhibitor group

HIF-1 $\alpha$ expression was measured after up-regulating KDM3A and it was found that HIF- $1 \alpha$ level was indeed enhanced by KDM3A overexpression (Fig. 4d). Furthermore, a two-way CO-IP test was performed and the result reflected that KDM3A and HIF- $1 \alpha$ were coprecipitated in A549 cells by anti-KDM3A antibody (Fig. 4e). It was suggested that HIF-1 $\alpha$ could bind to KDM3A.

\section{Up-regulating KDM3A or HIF-1a negates the overexpressed miR-449a-induced suppression on cellular growth in lung cancer}

Though we have confirmed the effects of miR-449a and KDM3A on lung cancer, and identified the targeting relation between miR-449a and KDM3A, as well as between KDM3A and HIF-1 $\alpha$, the internal mechanism of miR$449 \mathrm{a} / \mathrm{KDM} 3 \mathrm{~A} / \mathrm{HIF}-1 \alpha$ axis is not yet clear. Therefore, a co-transfection system was conducted: up-regulation of KDM3A or HIF-1 $\alpha$ was performed simultaneously with overexpression of miR-449a. Then, it was revealed that restoring KDM3A or HIF- $1 \alpha$ negated overexpressed miR-449a-induced effects on cellular growth in lung cancer (Fig. 5a-f). Moreover, miR-449a-mediated suppression on HIF- $1 \alpha$ expression was impaired by up-regulation of KDM3A (Additional file 2: Fig. S2e). Thus, it could be concluded that elevating KDM3A or HIF- $1 \alpha$ negated up-regulated miR-449a-induced suppression on cellular growth in lung cancer.

\section{Restoring miR-449a impairs tumorigenesis in vivo in lung cancer}

A549 cells that had been transfected with the plasmids were injected into mice to study whether miR-449a/

(See figure on next page.)

Fig. 3 Elevating KDM3A promotes cellular aggression in lung cancer while down-regulating KDM3A has the opposite effects. a CCK-8 assay analyzed A549 cell proliferation after interference with KDM3A; b Flow cytometry analyzed A549 cell cycle after interference with KDM3A; c Western blot analyzed the expression of apoptosis-related proteins in A549 cells after interference with KDM3A; d Flow cytometry analyzed A549 cell apoptosis after interference with KDM3A; e Transwell assay analyzed A549 cell migration after interference with KDM3A; f Transwell assay analyzed A549 cell invasion after interference with KDM3A; The data from three independent experiments were expressed as mean \pm standard deviation. ${ }^{*} P<0.05$ compared with the oe-NC group. $\# P<0.05$ compared with the sh-NC group 


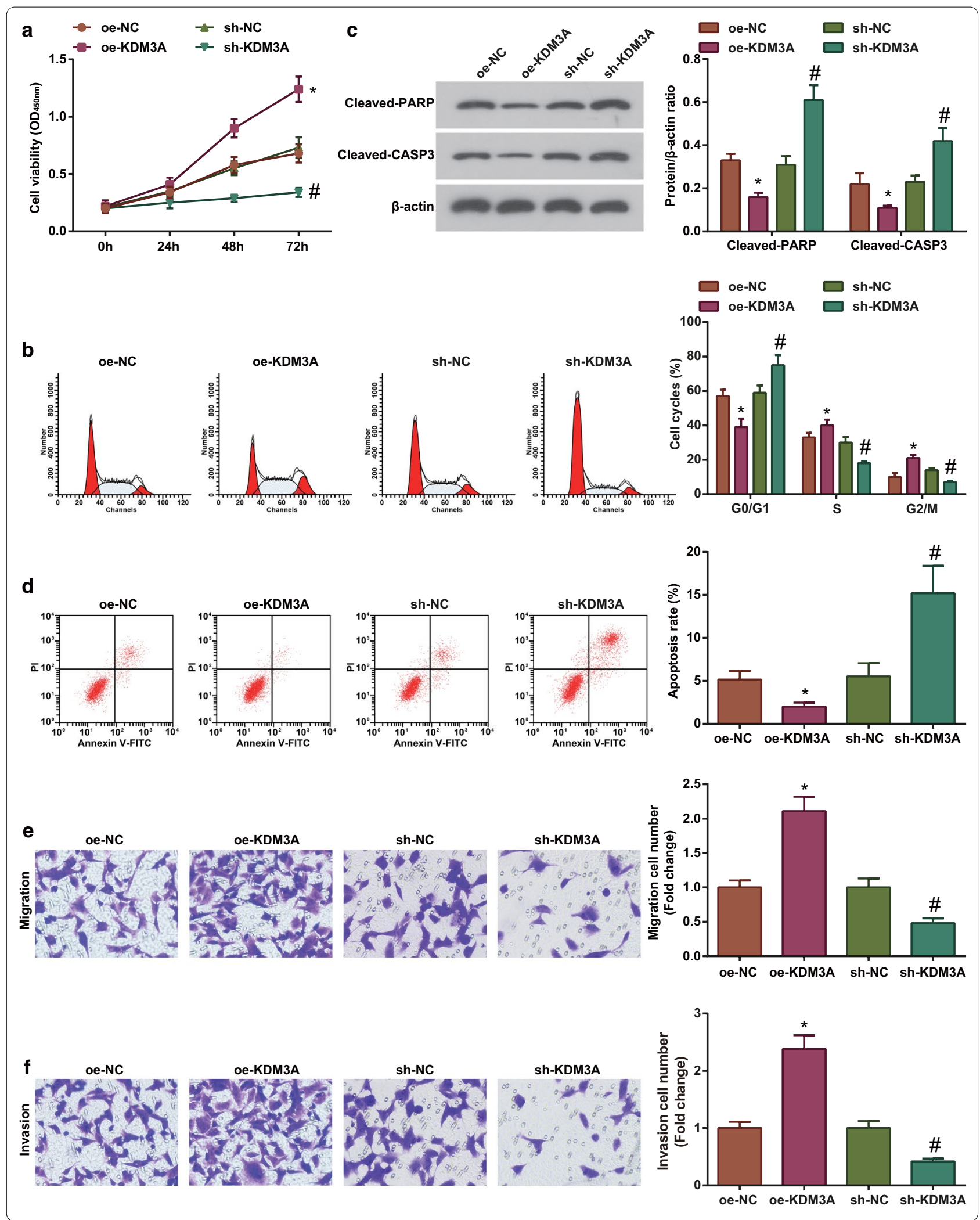



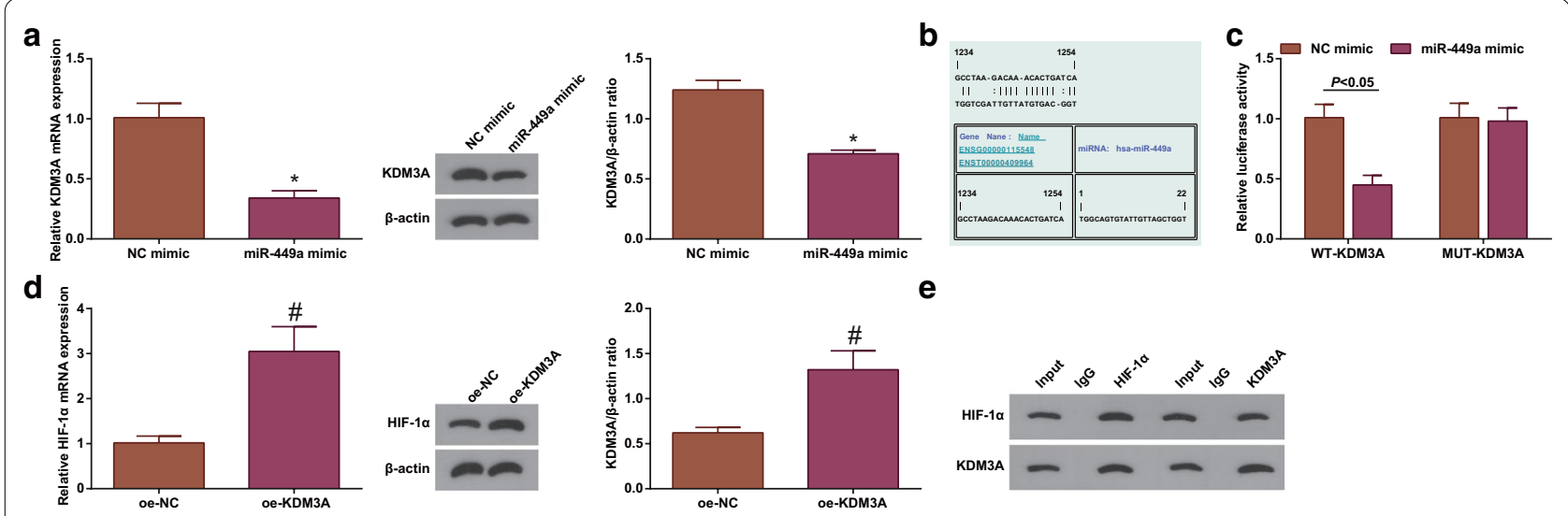

Fig. 4 miR-449a interacts with KDM3A; HIF-1 a could bind with KDM3A. a RT-qPCR and Western blot analyzed KDM3A expression in cells after overexpression of miR-449a; $\mathbf{b}$ Bioinformatics website predicted the binding sites of miR-449a and KDM3A; $\mathbf{c}$ Luciferase reporter gene experiment verified the relation between miR-449a and KDM3A; $\mathbf{d}$ RT-qPCR and Western blot analyzed HIF-1 a expression in cells after overexpression of KDM3A; e. CO-IP assay analyzed the interaction between KDM3A and HIF-1 ; The data from three independent experiments were expressed as mean \pm standard deviation. ${ }^{*} P<0.05$ compared with the the NC-mimic group; $\# P<0.05$ compared with the the oe-NC group

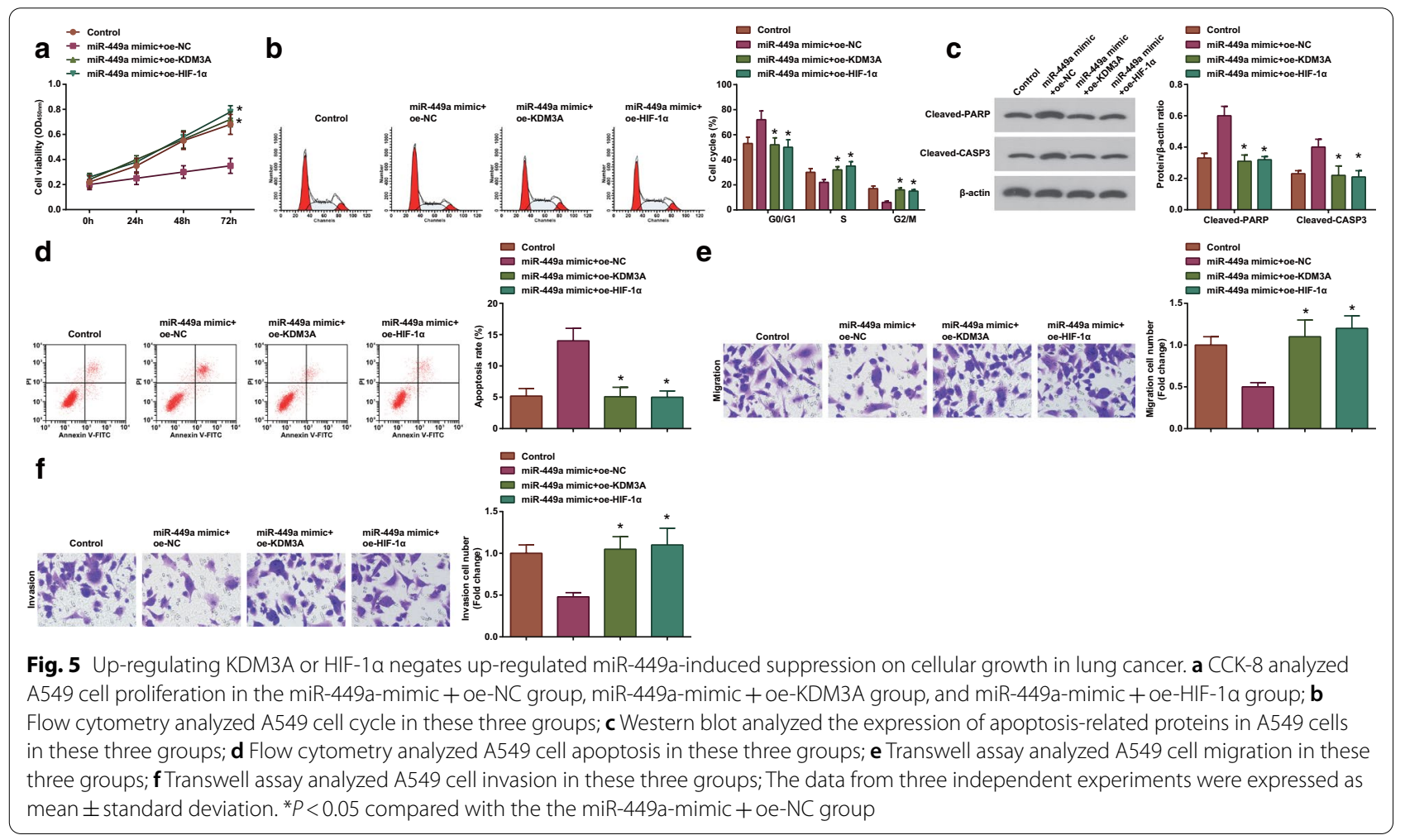

KDM3A/HIF-1 $\alpha$ axis regulated lung cancer progression in vivo. It was measured that up-regulating miR-449a decreased tumor volume and weight. However, KDM3A overexpression impaired such anti-tumor effects of miR449a on mice with lung cancer (Fig. 6a, b). In addition, miR-449a expression was increased whereas KDM3A and HIF- $1 \alpha$ levels were decreased in xenografted tumors treated with up-regulated miR-449a, which were reversed by enhanced expression of KDM3A (Fig. 6c-e). Shortly, miR-449a/KDM3A/HIF- $1 \alpha$ axis regulated lung cancer tumor growth in vivo. 


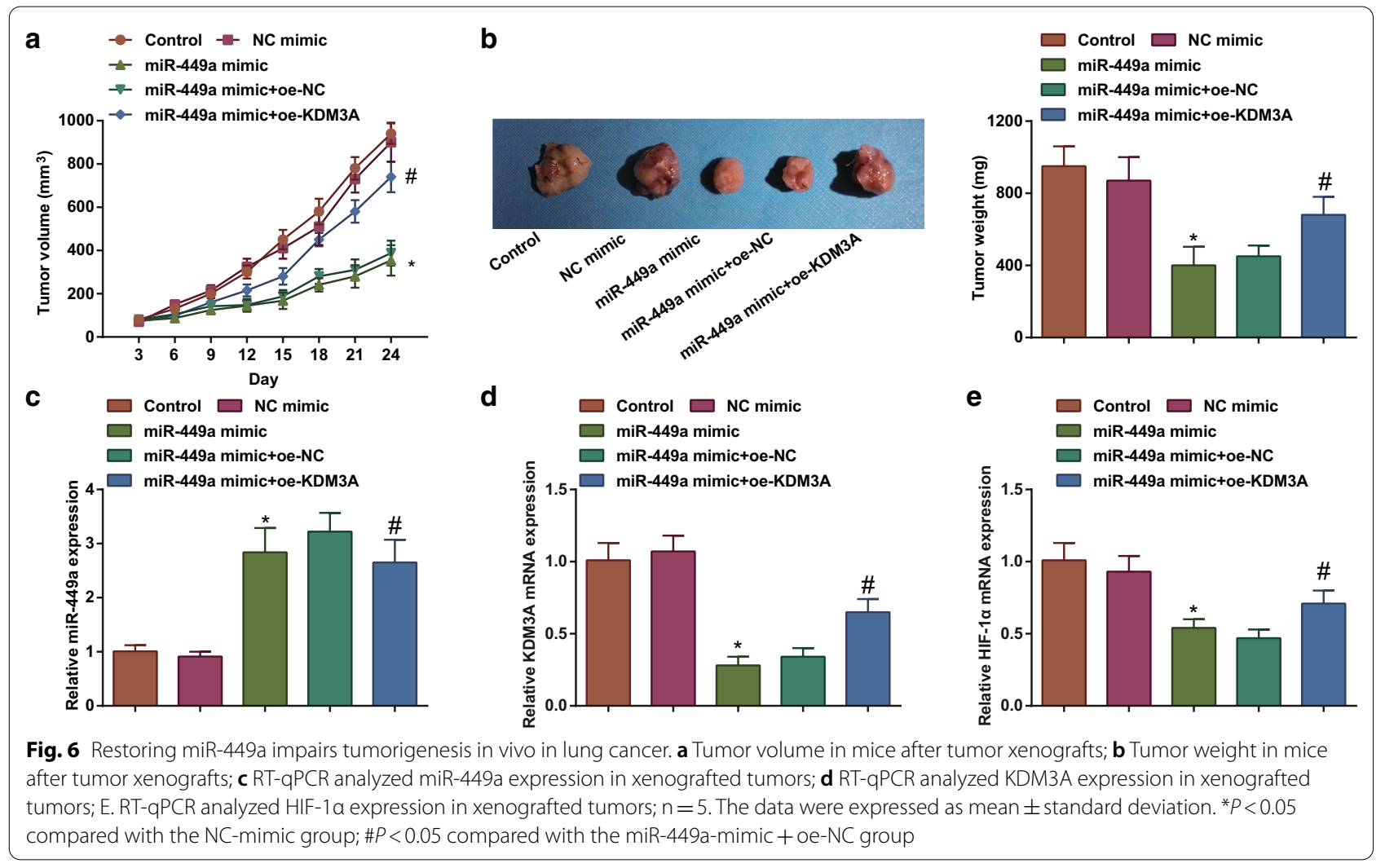

\section{Discussion}

Lung cancer remains the major cancer type in male and female with enigmatic pathogenesis [34]. In the present paper, a part of the underlying mechanism in lung cancer was explained from the axis of miR-449a/KDM3A/ HIF- $1 \alpha$. We determined a low expression of miR-449a in lung cancer and further figured out that over-expressing miR-449a delayed while suppressing miR-449a accelerated lung cancer development in vitro and in vivo, which was related to its negative regulation on HIF-1 $\alpha$. Besides, KDM3A, validated as the target of miR-449a, was overexpressed in lung cancer and promoted cellular aggression. Collectively, miR-449a depressed KDM3A to incur tumor activities in lung cancer through down-regulating HIF- $1 \alpha$.

miR-449a has been regarded as a tumor suppressor in cancers, whose functional overexpression could hamper cancer cell growth. The repressed level of miR-449a is also detected in NSCLC, and up-regulated miR-449a can damage proliferation, migration and invasion of cancer cells whereas down-regulated miR-449a functions in an opposite way [7]. As suggested in a late research, the proliferative and anti-apoptotic behaviors of lung cancer cells are limited by enhanced miR-449a, and greatly impaired by ultrasound-microbubble-mediated miR-449a [8]. The overexpressed miR-449a has been witnessed to negatively mediate A aisintegrin and metalloproteinases 10, thereby weakening the migratory and invasive capacities of NSCLC cells [35]. Through targeting high mobility group box 1, miR-449a sets the obstacle for NSCLC cells to proliferate, migrate and invade in vitro and to form tumors in vivo [18]. Further proved by a published report, lung cancer cells containing up-regulated miR-449a behave less aggressively and mainly arrest in G1/G0 phase [36]. Transient up-regulation of miR-449a in lung cancer cells functions to promote cell senescence and that in xenograted tumors depresses tumor growth, which is related the regulatory mechanism with E2F transcription factor 3 [17]. From those previous reports, it was confirmed that miR-449a was repressive for lung cancer development.

Though KDM3A was studied as a target of miR-449a in the present study, their targeting relation has not been widely validated in other researches, which needs further verification in proceeding explorations. Increased level of KDM3A has been shown in lung cancer and artificially silencing KDM3A obtains the ability to damage cell proliferation and migration [29]. In a former research focusing on NSCLC, the aberrantly elevated KDM3A expression manifests in cancer tissues and knocking down KDM3A attributes to the suppression on cell biological functions [12]. Not limited to lung cancer, the contributory effects of KDM3A overexpression are 
detected in other cancers. Exampled by a recent report, the invasive ability of breast cancer cells manifesting inhibited KDM3A is impaired in hypoxia [37]. KDM3A level can be elevated by hypoxia in pancreatic cancer and the incremental expression of KDM3A could stimulate cancer development whereas its suppression had the opposite effects [38]. Of interest, KDM3A restoration is the base to acquire an anti-apoptotic phenotype of myeloma cell apoptosis in chronic hypoxia through accumulating HIF-1 $\alpha$ [39].

Widely investigated, the up-regulated HIF- $1 \alpha$ exhibits in lung cancer $[40,41]$. The down-regulated HIF- $1 \alpha$ in NSCLC cells is a part contributing to the repressive effects of methanol-ethyl acetate partitioned fraction from Magnolia grandiflora on tumor cell invasion and migration [42]. HIF-1 $\alpha$ down-regulation is capable of preventing cellular growth and stimulating cell apoptosis in NSCLC [43]. HIF-1 $\alpha$ level can be suppressed by up-regulating miR-199a, thereafter devoting to NSCLC cell proliferation suppression [44]. The depletion of long non-coding RNA cancer susceptibility 9 suppressively modifies the proliferation, invasion and migration of lung cancer, which is connected with its positive feedback loop with HIF-1 $\alpha$ [45].

\section{Conclusion}

On the whole, it is summarized that miR-449a interacts with KDM3A/HIF- $1 \alpha$ axis, and performs anti-tumor effects on lung cancer. From the axis of miR-449a/ KDM3A/HIF- $1 \alpha$, the molecular mechanism of lung cancer has been further comprehended, and this axis may alight the potentials to treat lung cancer. Limitations to relative small experimental scale ask for further confirmation of the results in a larger study size.

\section{Abbreviations \\ miR: MicroRNA; KDM3A/HIF-1a: Lysine demethylase 3A/hypoxia-induced factor-1a; NSCLC: Non-small cell lung cancer; miRNAs: MicroRNAs; FBS: Fetal bovine serum; CCK: Cell counting kit; RT-qPCR: Reverse transcription quantita- tive polymerase chain reaction; GAPDH: Glyceraldehyde-3-phosphate dehy- drogenase; 3'UTR: 3'Untranslated region; Co-IP: Co-immunoprecipitation.}

\section{Supplementary Information}

The online version contains supplementary material available at https://doi. org/10.1186/s12967-021-02881-8.

\footnotetext{
Additional file 1: Figure S1 RT-qPCR and Western blot analysis of miR449a and KDM3A expression. a RT-qPCR analyzed miR-449a expression in lung cancer tissues and normal tissues, $n=80 ; \mathbf{b}$ RT-qPCR analyzed miR449a expression in normal human lung epithelial cell lines BEAS-2B and lung cancer cell lines (A549, H1299 and H460); c RT-qPCR analyzed miR449a expression in A549 cells after overexpression of miR-449a; $\mathbf{d}$ RT-qPCR analyzed miR-449a expression in A549 cells after inhibiting miR-449a; $\mathbf{e}$ RT-qPCR and Western blot analyzed KDM3A expression in lung cancer tissues and normal tissues, $n=80 ; \mathbf{f}$. RT-qPCR and Western blot analyzed
}

KDM3A expression in normal human lung epithelal cell line BEAS-2B and lung cancer cell lines (A549, H1299 and H460); g RT-qPCR and Western blot analyzed KDM3A expression in A549 cells after interference with KDM3A; The data from three independent experiments were expressed as mean \pm standard deviation. ${ }^{*} P<0.05$ compared with the oe-NC group. \# $P<0.05$ compared with the sh-NC group.

Additional file 2: Figure S2 RT-qPCR and Western blot analysis of HIF-1a expression. a RT-qPCR analyzed HIF-1 a expression in lung cancer tissues and normal tissues, $\mathrm{n}=80$; $\mathbf{b}$ RT-qPCR analyzed HIF-1 a expression in normal human lung epithelial cell line BEAS-2B and lung cancer cell lines (A549, H1299 and H460); c RT-qPCR and Western blot analyzed HIF-1a expression in A549 cells after overexpression of miR-449a; d RT-qPCR and Western blot analyzed HIF-1a expression in cells after inhibiting miR-449a; e RT-qPCR and Western blot analyzed HIF-1a expression in these three groups; The data from three independent experiments were expressed as mean \pm standard deviation. ${ }^{*} P<0.05$ compared with the NC-mimic group. $\# P<0.05$ compared with the NC-inhibitor group. \& $P<0.05$ compared with the the miR-449a-mimic + oe-NC group.

Additional file 3: Figure $\mathbf{S 3}$ Overexpression of miR-449a suppresses the growht of H1299 cells. a RT-qPCR analyzed miR-449a expression in H1299 cells after overexpression of miR-449a; b CCK-8 assay analyzed H1299 cell proliferation after overexpression of miR-449a; c Flow cytometry analyzed H1299 cell cycle after overexpression of miR-449a; $\mathbf{d}$ Western blot analyzed the expression of apoptosis-related proteins in $\mathrm{H} 1299$ cells after overexpression of miR-449a; e Flow cytometry analyzed H1299 cell apoptosis after overexpression of miR-449a; f Transwell assay analyzed H1299 cell migration after overexpression of miR-449a; $\mathbf{g}$ Transwell assay analyzed H1299 cell invasion after overexpression of miR-449a; The data from three independent experiments were expressed as mean \pm standard deviation. *indicates $P<0.05$ compared with the NC-mimic group.

\section{Acknowledgements}

We would like to acknowledge the reviewers for their helpful comments on this paper.

\section{Authors' contributions}

FL, BZ finished study design, SH, PC, KK finished experimental studies, SH, PH, YD finished data analysis, SH finished manuscript editing. All authors read and approved the final manuscript.

Funding

None.

Availability of data and materials

Not applicable.

\section{Declarations}

\section{Ethics approval and consent to participate}

This study was approved and supervised by the animal ethics committee of Tongji Hospital, Tongji Medical College, Huazhong University of Science and Technology. The treatment of animals in all experiments conforms to the ethical standards of experimental animals.

\section{Consent for publication}

Not applicable.

\section{Competing interests}

The authors declare that they have no conflicts of interest.

Received: 29 November 2020 Accepted: 10 May 2021

Published online: 27 May 2021 


\section{References}

1. Zhang T, et al. The Role of RASSF1 Methylation in Lung Carcinoma. Adv Exp Med Biol. 2020;1255:99-108.

2. Xu R, et al. The momentous role of N6-methyladenosine in lung cancer. J Cell Physiol. 2020;236:3244.

3. Vavala T, et al. An examination of two dichotomies: Women with lung cancer and living with lung cancer as a chronic disease. Respirology. 2020;25(Suppl 2):24-36.

4. Yin Z, et al. Immunoregulatory roles of extracellular vesicles and associated therapeutic applications in lung cancer. Front Immunol. 2020;11:2024

5. Zhao L, et al. Multiple primary lung cancers: a new challenge in the era of precision medicine. Cancer Manag Res. 2020;12:10361-74.

6. Santos RM, Moreno C, Zhang WC. Non-coding RNAs in lung tumor initiation and progression. Int J Mol Sci. 2020;21(8):2774.

7. Pang $\mathrm{L}$, et al. Long noncoding RNA SNHG7 contributes to cell proliferation, migration, invasion and epithelial to mesenchymal transition in nonsmall cell lung cancer by regulating miR-449a/TGIF2 axis. Thorac Cancer. 2020;11(2):264-76.

8. Meng L, et al. Ultrasound-microbubbles-mediated microRNA-449a inhibits lung cancer cell growth via the regulation of Notch1. Onco Targets Ther. 2019:12:7437-50.

9. Bach DH, et al. Targeting nicotinamide N-methyltransferase and miR-449a in EGFR-TKI-resistant non-small-cell lung cancer cells. Mol Ther Nucleic Acids. 2018;11:455-67.

10. Li L, et al. miR-449a Suppresses LDHA-mediated glycolysis to enhance the sensitivity of non-small cell lung cancer cells to ionizing radiation. Oncol Res. 2018;26(4):547-56.

11. Cho HS, et al. The JmjC domain-containing histone demethylase KDM3A is a positive regulator of the G1/S transition in cancer cells via transcriptional regulation of the HOXA1 gene. Int J Cancer. 2012;131(3):E179-89.

12. Zhan M, et al. JMJD1A promotes tumorigenesis and forms a feedback loop with EZH2/let-7c in NSCLC cells. Tumour Biol. 2016;37(8):11237-47.

13. Li Y, et al. KDM3A promotes inhibitory cytokines secretion by participating in TLR4 regulation of Foxp3 transcription in lung adenocarcinoma cells. Oncol Lett. 2017;13(5):3529-37.

14. Guo X, et al. Ascorbate antagonizes nickel ion to regulate JMJD1A expression in kidney cancer cells. Acta Biochim Biophys Sin (Shanghai). 2012;44(4):330-8.

15. Huang $X$, Zhou W, Zhang $Y$. Transcription factor $Y Y 1$ enhances the stemness of lung cancer cells by stabilizing hypoxia factor HIF-1alpha under a hypoxic microenvironment. Environ Toxicol. 2020;36:114.

16. Pandey $\mathrm{N}$, et al. Allicin overcomes hypoxia mediated cisplatin resistance in lung cancer cells through ROS mediated cell death pathway and by suppressing hypoxia inducible factors. Cell Physiol Biochem. 2020;54(4):748-66.

17. Ren XS, et al. Tumor-suppressive microRNA-449a induces growth arrest and senescence by targeting E2F3 in human lung cancer cells. Cancer Lett. 2014;344(2):195-203.

18. Wu D, et al. miR-449a Suppresses tumor growth, migration, and invasion in non-small cell lung cancer by targeting a HMGB1-mediated NF-kappaB signaling pathway. Oncol Res. 2019;27(2):227-35.

19. Song $N$, et al. MicroRNA-138-5p suppresses non-small cell lung cancer cells by targeting PD-L1/PD-1 to regulate tumor microenvironment. Front Cell Dev Biol. 2020:8:540.

20. Song LN, et al. Hsa_circ_0003998 promotes epithelial to mesenchymal transition of hepatocellular carcinoma by sponging miR-143-3p and PCBP1. J Exp Clin Cancer Res. 2020;39(1):114.

21. Ye $X, L v H$. MicroRNA-519d-3p inhibits cell proliferation and migration by targeting TROAP in colorectal cancer. Biomed Pharmacother. 2018;105:879-86

22. Zhang S, et al. MEF2activated long noncoding RNA PCGEM1 promotes cell proliferation in hormonerefractory prostate cancer through downregulation of miR148a. Mol Med Rep. 2018;18(1):202-8.

23. Li X, et al. MicroRNA-449a inhibits proliferation and induces apoptosis by directly repressing E2F3 in gastric cancer. Cell Physiol Biochem. 2015;35(5):2033-42

24. Gu J, et al. Effect of the LncRNA GAS5-MiR-23a-ATG3 axis in regulating autophagy in patients with breast cancer. Cell Physiol Biochem. 2018;48(1):194-207.
25. Robinson TE, et al. Mucus plugging, air trapping, and bronchiectasis are important outcome measures in assessing progressive childhood cystic fibrosis lung disease. Pediatr Pulmonol. 2020:55(4):929-38.

26. He C, et al. Lysine demethylase KDM3A regulates nanophotonic hyperthermia resistance generated by 2D silicene in breast cancer. Biomaterials. 2020;255:120181.

27. Xie F, et al. MiR-143-3p suppresses tumorigenesis in pancreatic ductal adenocarcinoma by targeting KRAS. Biomed Pharmacother. 2019:119:109424.

28. Jeon HS, et al. Combining microRNA-449a/b with a HDAC inhibitor has a synergistic effect on growth arrest in lung cancer. Lung Cancer. 2012;76(2):171-6.

29. Li Z, et al. Epigenetic regulation of lung cancer cell proliferation and migration by the chromatin remodeling protein BRG1. Oncogenesis. 2019;8(11):66

30. Chang HL, Lin JC. SRSF1 and RBM4 differentially modulate the oncogenic effect of HIF-1alpha in lung cancer cells through alternative splicing mechanism. Biochim Biophys Acta Mol Cell Res. 2019;1866(12):118550.

31. Yoo J, et al. Advances in histone demethylase KDM3A as a cancer therapeutic target. Cancers (Basel). 2020;12(5):1098

32. Mimura I, Nangaku M. Epigenetics in kidney diseases. Rinsho Byori. 2014;62(2):180-9.

33. Mimura l, et al. Dynamic change of chromatin conformation in response to hypoxia enhances the expression of GLUT3 (SLC2A3) by cooperative interaction of hypoxia-inducible factor 1 and KDM3A. Mol Cell Biol. 2012;32(15):3018-32.

34. Mederos N, et al. Gender-specific aspects of epidemiology, molecular genetics and outcome: lung cancer. ESMO Open. 2020;5(Suppl 4):e000796.

35. Meng $\mathrm{H}$, et al. MiR-449a regulates the cell migration and invasion of human non-small cell lung carcinoma by targeting ADAM10. Onco Targets Ther. 2019;12:3829-38.

36. You J, et al. MicroRNA-449a inhibits cell growth in lung cancer and regulates long noncoding RNA nuclear enriched abundant transcript 1. Indian J Cancer. 2014;51(Suppl 3):e77-81.

37. Ahn $\mathrm{HJ}$, et al. KDM3A regulates Slug expression to promote the invasion of MCF7 breast cancer cells in hypoxia. Oncol Lett. 2020;20(6):335.

38. Dandawate $P$, et al. The histone demethylase KDM3A, increased in human pancreatic tumors, regulates expression of DCLK1 and promotes tumorigenesis in mice. Gastroenterology. 2019;157(6):1646-1659 e11.

39. Ikeda S, et al. Hypoxia-inducible KDM3A addiction in multiple myeloma. Blood Adv. 2018;2(4):323-34.

40. Huang $B$, et al. The role of monoamine oxidase A in HPV-16 E7-induced epithelial-mesenchymal transition and HIF-1alpha protein accumulation in non-small cell lung cancer cells. Int J Biol Sci. 2020;16(14):2692-703.

41. Yin X, et al. CHCHD2 is a potential prognostic factor for NSCLC and is associated with HIF-1a expression. BMC Pulm Med. 2020;20(1):40.

42. $\mathrm{Ma} \mathrm{H}$, et al. Anti-cancer effects of methanol-ethyl acetate partitioned fraction from Magnolia grandiflora in human non-small cell lung cancer H1975 cells. J Bioenerg Biomembr. 2020;52(3):175-83.

43. $\mathrm{Hu} \mathrm{H}$, et al. YC-1 potentiates the antitumor activity of gefitinib by inhibiting HIF-1alpha and promoting the endocytic trafficking and degradation of EGFR in gefitinib-resistant non-small-cell lung cancer cells. Eur J Pharmacol. 2020;874:172961.

44. Wang LM, et al. Influence of miR-199a on rats with non-small cell lung cancer via regulating the HIF-1alpha/NEGF signaling pathway. Eur Rev Med Pharmacol Sci. 2019;23(23):10363-9.

45. Jin Y, et al. Long non-coding RNA CASC9 And HIF-1alpha form a positive feedback loop to facilitate cell proliferation and metastasis in lung cancer Onco Targets Ther. 2019;12:9017-27.

\section{Publisher's Note}

Springer Nature remains neutral with regard to jurisdictional claims in published maps and institutional affiliations. 